\title{
Validation of the event generator mode in the PHITS code and its application
}

\author{
Yosuke Iwamoto $^{1, a}$, Koji Niita ${ }^{2}$, Yukio Sakamoto ${ }^{1}$, Tatsuhiko Sato ${ }^{1}$, and Norihiro Matsuda ${ }^{1}$ \\ 1 Japan Atomic Energy Agency (JAEA), 2-4 Shirakata, Tokai-mura, Naka-gun, Ibaraki 319-1195, Japan \\ 2 Research Organization for Information Science and Technology (RIST), 2-4 Shirakata, Tokai-mura, Naka-gun, Ibaraki 319-1195, Japan
}

\begin{abstract}
The "Event Generator Mode" has been introduced into the PHITS code in the treatment of low energy transport phenomena. In this mode, the evaluated nuclear data for neutrons and a special evaporation model are combined so as to trace all correlations of ejectiles keeping the energy and the momentum conservation in a collision. We have calculated the double differential cross section, the kerma coefficient and the displacement cross section by the event generator mode of PHITS, and compared them with the evaluated nuclear data for the validation of the mode in neutron-induced reactions under $30 \mathrm{MeV}$. As results, all calculated results by the event generator mode reproduce the evaluated nuclear data well below $20 \mathrm{MeV}$. As an application of this mode, we indicate a new approach to the deposit energy distribution for the thermal neutron transport and the averaged quality factor for neutrons.
\end{abstract}

\section{Introduction}

The concept of the event generator is rather popular with the Monte Carlo simulation code for high energy particles. In the event generator mode, the conservation law on the energy and the momentum is sustained in each event. Recently, the correlated quantities such as event-by-event distribution of deposit energy even in low energy fields are often required for microdosimetric estimations of irradiation effects in biology and semiconductors. For these requirements, the "Event Generator Mode" has been introduced into the PHITS code [1] in the transport of low energy neutrons. In this mode, the evaluated nuclear data for neutrons and a special evaporation model are combined so as to trace all correlations of ejectiles keeping the energy and the momentum conservation in a collision. This mode enables us to calculate the correlated quantities mentioned above, and also to estimate the kerma coefficient and the displacement cross section data without additional evaluated cross section libraries.

In this work, we have calculated particle production cross sections, the kerma coefficient and the displacement cross section by the event generator mode of PHITS, and compared them with the evaluated nuclear data for the validation of the mode in neutron-induced reactions under $30 \mathrm{MeV}$. As an application of this mode, we will suggest a new approach to the deposit energy distribution for the thermal neutron transport and the averaged quality factor for radiation protection against low energy neutron exposure.

\section{Method of calculations}

A brief flowchart of the Event Generator Mode is shown in figure 1. A detail is presented in the other paper of these proceedings [2]. The evaluated neutron data of the total cross section, the channel cross section, i.e., capture, elastic and $\left(\mathrm{n}, \mathrm{n}^{\prime}\right),(\mathrm{n}, \mathrm{Nn})$ cross section are used for branching the

${ }^{a}$ Presenting author, e-mail: iwamoto.yosuke@jaea.go.jp

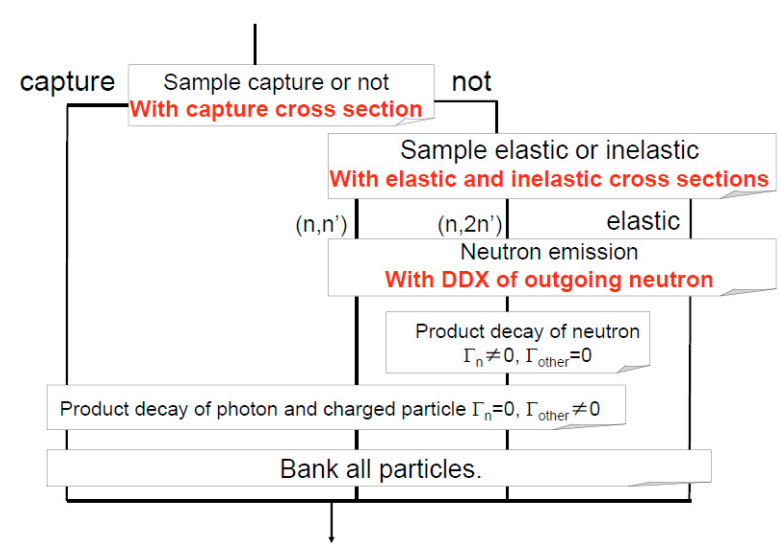

Fig. 1. A brief flowchart of the event generator mode.

reactions, and the inclusive double differential cross section of outgoing neutrons are used for the spectrum of the first emitted neutron.

For a capture reaction, the excitation energy and momentum of captured nucleus is determined uniquely from the incident energy of neutron and target nucleus. A special statistical decay model [3] without the decay width of neutron is applied to this excited nucleus. Then all information of ejectiles, in this case, charged particles, photon and residual nucleus can be determined.

For an elastic reaction, the scattering angle of outgoing neutron is determined according to the nuclear data. By the kinematics of this elastic channel, the energy of neutron and the momentum of the residual nucleus can be determined uniquely.

For $\left(n, n^{\prime}\right)$ reactions, the momentun of outgoing neutron is determined according to the double differential cross section of the nuclear data. By the kinematics of this emission, the excitation energy and momentum of the residual nucleus is determined uniquely. Next, the statistical decay process without neutron decay width is also applied. 
Finally, for $\left(\mathrm{n}, \mathrm{Nn}^{\prime}\right)$ reaction, a similar way as in the $\left(\mathrm{n}, \mathrm{n}^{\prime}\right)$ case is applied, but after one neutron emission, the statistical decay process by only neutron channels is adopted until $\mathrm{N}$ nucleons are emitted. After this, the statistical decay process without neutron decay width is applied.

By these processes, we can treat a low energy neutron collision as an "event", which means the energy and momentum are conserved in an event. Therefore, by this mode, we can extract any information, e.g., the kinetic energy distribution of the residual nuclei, two-particle correlation, etc. in the transport calculation of PHITS.

\section{Validation of the event generator mode}

In this section, we indicate the validation of the event generator mode by showing the comparisons of the double differential cross section, the kerma coefficient and the displacement cross section.

\subsection{Double differential cross section}

For the evaluation of the kerma coefficient, an important thing is the kinetic energy distribution of the charged particles and the residual nuclei produced by a collision. Figure 2 shows the double differential cross section of alpha for the neutron induced reaction at $14 \mathrm{MeV}$ on carbon at 19 degree. The point shows experimental data by Haight et al. [4]. A peak at the high energy region indicates $(\mathrm{n}, \alpha)$ reaction and that at low energy indicates $\left(\mathrm{n}, \mathrm{n}^{\prime} 3 \alpha\right)$ reaction respectively [5]. Both results calculated by event generator mode with LA150 [6] (solid line) and JENDL-HE [7] (dashed line) neutron data files reproduce these reactions and give good agreements with experimental data.

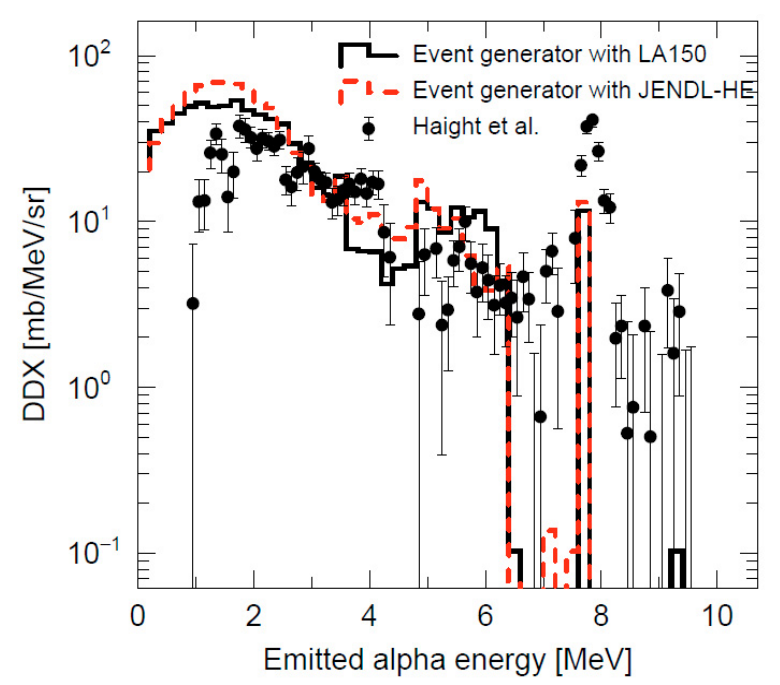

Fig. 2. The double differential cross section of alpha for neutron induced reaction at $14 \mathrm{MeV}$ on ${ }^{12} \mathrm{C}$ at 19 degree.

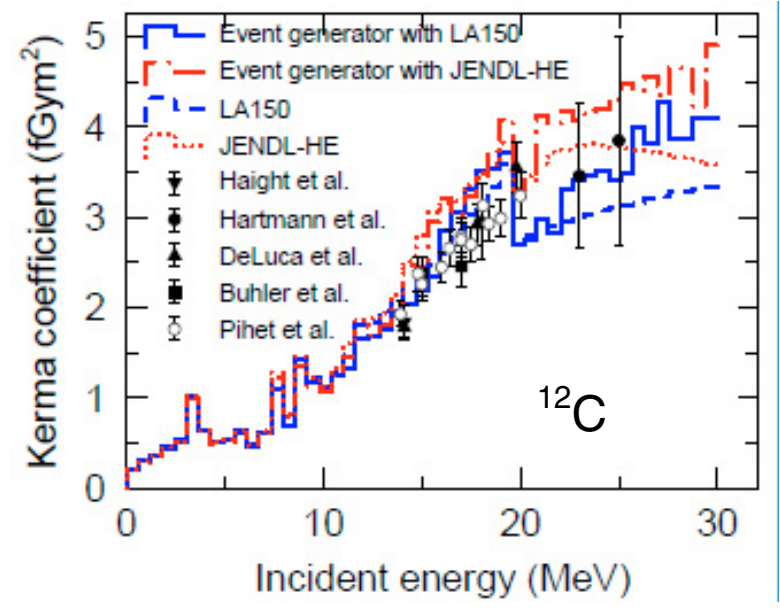

Fig. 3. Kerma coefficients as a function of the incident neutron energy on ${ }^{12} \mathrm{C}$.

\subsection{Kerma coefficient}

The kerma coefficient, which is the sum of the kinetic energy of the charged particles and the recoil nuclei from neutron induced reaction, can be calculated from the energy loss of all charged particles and recoil nuclei in the event generator mode of PHITS. Figure 3 shows the kerma coefficient as a function of the incident neutron energy on ${ }^{12} \mathrm{C}$.

Solid and dot-dashed lines are the results of the event generator mode with neutron data files of LA150 and JENDLHE, respectively. Dashed and dot lines show the kerma coefficients of the additional evaluated data included in LA150 and JENDL-HE, respectively. The measured values are taken from refs [8-12]. The results of the event generator mode are in agreement with the measurements and the additional evaluated nuclear data at neutron energies below $20 \mathrm{MeV}$. On the other hand, a large discontinuity by the event generator mode appears at $20 \mathrm{MeV}$. This might be mainly due to a lack of the neutron capture cross section in the neutron data files above $20 \mathrm{MeV}$. The kerma coefficients of the additional evaluated data also show discontinuity at $20 \mathrm{MeV}$. For this reason, the evaluated kerma data files have to be improved to connect smoothly at $20 \mathrm{MeV}$.

\subsection{Displacement cross section}

For the construction of a maintenance scenario of the strong neutron source in the accelerator facility, the life estimation of those structure materials becomes indispensable. Therefore, displacement per atom (DPA) value of each component is needed, since DPA is a major index of the radiation damage. For the estimation of DPA in the transport calculations, we need the displacement cross-section $\sigma_{D X}(E)$, which is defined by the displacement damage function for each charged particles and recoil nuclei produced by the collisions as a function of the kinetic energy of them. The kinetic energy distributions of them are calculated by the event generator mode of PHITS in event by event. 


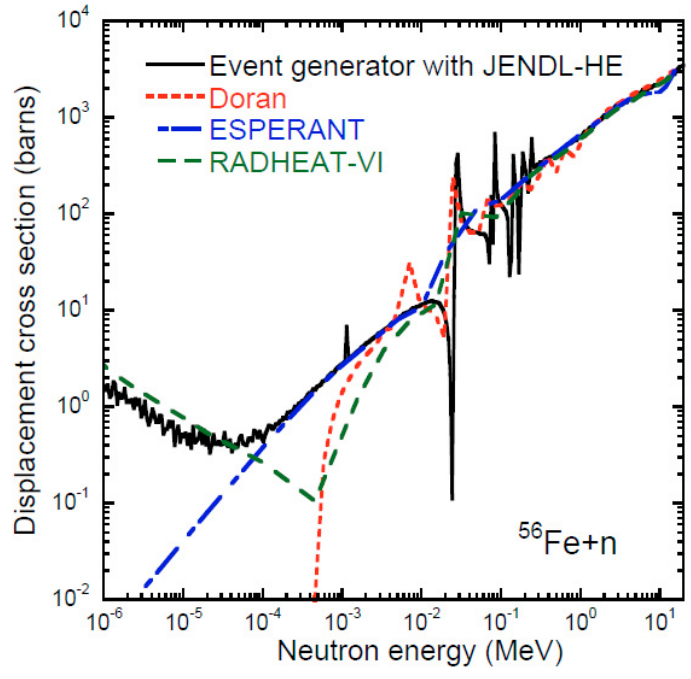

Fig. 4. Displacement cross sections as a function of the incident neutron energy on ${ }^{56} \mathrm{Fe}$.

Figure 4 shows the results of the displacement cross section for the neutron incident reaction on ${ }^{56} \mathrm{Fe}$. Values of Doran, ESPERAND and RADHEAT-VI are taken from ref. [16-18], respectively. All of the displacement cross sections in the neutron energy region above $10^{-3} \mathrm{MeV}$, where is in the elastic scattering region, are almost the same. For the energy region below $10^{-4} \mathrm{MeV}$, the results of the event generator mode increase as the incident neutron energy decreases. This is also seen in RASHEAT-VI result, since the event generator mode and RADHEAT-VI can treat the capture reaction.

\section{Application of the event generator mode}

Event generator mode can calculate the energy and linear energy transfer (LET) distributions of produced charged particles and recoil nuclei. In this section, we will show two applications related to these quantities.

\subsection{Deposit energy distribution for the ${ }^{10} \mathrm{~B}\left(\mathrm{n}_{\mathrm{th}} \alpha\right)^{7} \mathrm{Li}$ reaction}

The thermal neutron induced ${ }^{10} \mathrm{~B}\left(\mathrm{n}_{\text {th }}, \alpha\right){ }^{7} \mathrm{Li}$ reaction will be very important in the microdosimetric analysis for the boronneutron capture therapy. After the capture of thermal neutron by ${ }^{10} \mathrm{~B},{ }^{11} \mathrm{~B}$ compound nucleus is formed in the intermediate state. The primary decay mode for this system is alpha particle decay. For the decay, the residual lithium nucleus can be left in either its ground state ${ }^{7} \mathrm{Li}$ or in its first excited state ${ }^{7} \mathrm{Li}^{*}$ which decays by prompt gamma emission with $E_{\text {gamma }}=478.5 \mathrm{keV}$. In the first case, the alpha particle has the kinetic energy $\mathrm{T}\left(\alpha_{0}\right)=1.79 \mathrm{MeV}$, we call this 'alpha zero' $\alpha_{0}$ and in the second case, $\mathrm{T}\left(\alpha_{1}\right)=1.48 \mathrm{MeV}$, called 'alpha one' $\alpha_{1}$. In the event generator mode, the branching ratio ${ }^{10} \mathrm{~B}\left(n, \alpha_{1}\right) /{ }^{10} \mathrm{~B}\left(n, \alpha_{0}\right)$ is set to be 15 taken from ENDF/B-VI standard file. Figure 5 shows the energy distributions of produced alpha, ${ }^{7} \mathrm{Li}$ and gamma calculated by the event generator mode of PHITS.

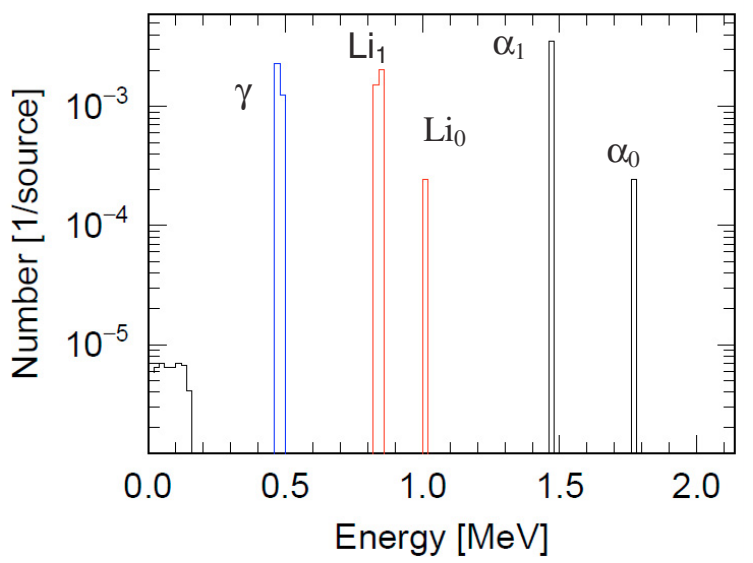

Fig. 5. Energy distribution of ${ }^{7} \mathrm{Li}$, alpha and gamma for the ${ }^{10} \mathrm{~B}\left(\mathrm{n}_{\mathrm{th}}, \alpha\right){ }^{7} \mathrm{Li}$ reaction.

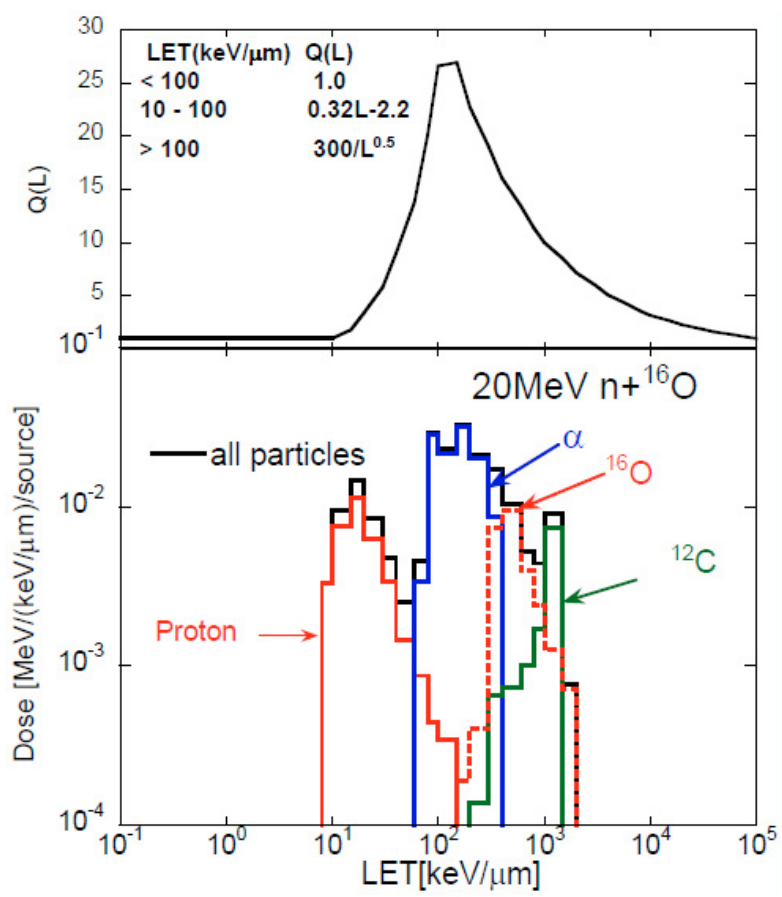

Fig. 6. The Q-L relation (upper panel) and dose spectra (lower panel) as a function of LET of the charged particles and recoil nuclei produced in the neutron induced reaction on ${ }^{16} \mathrm{O}$ at $20 \mathrm{MeV}$ calculated by the event generator mode of PHITS by using JENDL3.2 neutron data.

From this result, we can confirm the accuracy of the energy distribution of charged particles, recoil nuclei and gamma produced in low energy neutron reactions. Thus the event generator mode of PHITS would be applied more extensively to the microdosimetric analysis of the biological effects by thermal neutrons in future.

\subsection{The averaged quality factor for neutrons}

The quality factor $Q$ is important value for radiation protection and dosimetry. $Q$ at a point in human tissue is then given by 


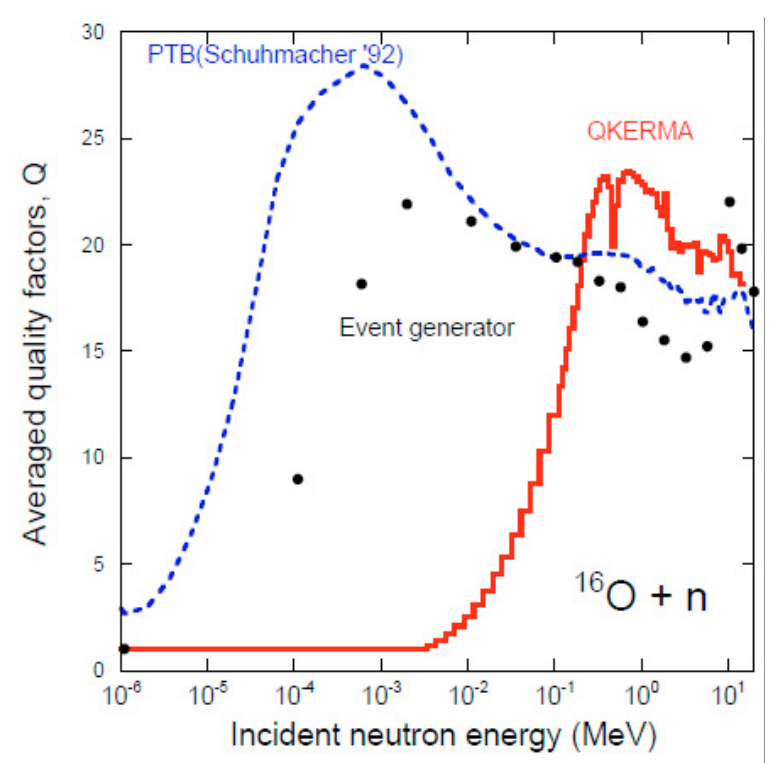

Fig. 7. Averaged quality factors. The full line is the results of QKERMA [22] and the dashed line denotes PTB [23], while the squares are the results of the event generator mode of PHITS.

ICRP60 [19]

$$
Q=\frac{1}{D} \int_{0}^{\infty} Q(L) D_{L} d L
$$

where $Q(L)$ the quality factor to LET specified by ICRP60 and $D_{L}$ the distribution of absorbed dose in $L$ at the point of an interest. As the first step, we evaluated the averaged quality factor for neutrons at i-th element of human tissue $Q_{i}$ by using dose spectra.

$$
Q_{i}=\frac{\int Q(L) D_{i}(L) d L}{\int D_{i}(L) d L},
$$

where $D_{i}(L)$ the dose spectra at i-th element of human tissue in $L$ by neutron incidence. Figure 6 shows the Q-L relation [19] (upper panel) and the dose spectra (lower panel) as a function of LET of the charged particles and recoil nuclei produced in the neutron induced reaction on ${ }^{16} \mathrm{O}$ at $20 \mathrm{MeV}$ calculated by the event generator mode of PHITS by using JENDL-3.2 neutron data [20].

The conversion from the energy to LET in water was done by SPAR code [21] in PHITS. The dose spectra include mainly proton, $\alpha,{ }^{12} \mathrm{C}$ and ${ }^{16} \mathrm{O}$ particles. The $\alpha$ particle especially contributes to the average quality factor with relation to $\mathrm{Q}(\mathrm{L})$. Figure 7 compares the averaged quality factors calculated by equation (2) with the results of QKERMA [22] and PTB [23]. The shape of our result is somewhat similar to the result of PTB and is significantly different from the QKERMA below $10^{-1} \mathrm{MeV}$. As a next step, we will evaluate the averaged quality factor for all elements of human tissues and compare other evaluated values.

\section{Summary}

The event generator mode of PHITS has been developed to conserve the energy and momentum in an event for the low energy transport phenomena. To investigate the validation of the event generator mode in the energy region below $30 \mathrm{MeV}$, the double differential cross section, the kerma coefficient and the displacement cross section were calculated and compared with the evaluated nuclear data and experimental data. As results, all calculated results by the event generator mode reproduce well the data below $20 \mathrm{MeV}$. As applications of this mode, we indicated new calculations, the deposit energy distributions of charge particles for the ${ }^{10} \mathrm{~B}\left(\mathrm{n}_{\mathrm{th}}, \alpha\right){ }^{7} \mathrm{Li}$ reaction and the averaged quality factor for neutron induced reaction on ${ }^{16} \mathrm{O}$. In future, the event generator mode of PHITS will be applied to the analysis of the biological effects, the single setup error of semiconductor, and to many other fields particularly to microdosimetry field.

\section{References}

1. H. Iwase, K. Niita, T. Nakamura, J. Nucl. Sci. Technol. 39, 1142 (2002).

2. K. Niita, Y. Iwamoto, T. Sato, H. Iwase, N. Matsuda, Y. Sakamoto, H. Nakashima (these proceedings).

3. S. Furihata, Nucl. Instrum. Meth. B 343, 197 (2005).

4. Y. Watanabe, T. Fukahori, K. Kosako et al., AIP Conf. Proc. 769, 1646 (2005).

5. H.M. Gerstenberg, R.S. Caswell, J.J. Coyne, Radiat. Prot. Dosim. 23, 41 (1988).

6. M.B. Chadwick et al., Nucl. Sci. Eng. 131, 293 (1999).

7. Nuclear data for neutron and proton radiotherapy and radiation protection, ICRU Report 63 (1999).

8. R.C. Haight, S.M. Grimes, R.G. Johnson, H.H. Barschall, Nucl. Sci. Eng. 87, 41 (1984).

9. C.L. Hartmann, P.M. Deluca Jr., D.W. Pearson, Radiat. Prot. Dosim. 44, 25 (1992).

10. P.M. Deluca Jr., H.H. Barschall, M. Burhoe, R.C. Haight, Nucl. Sci. Eng. 94, 192 (1986).

11. G. Buhler, H.G. Menzel, H. Schuhmacher, G. Dietze, Radiat. Prot. Dosim. 13, 13 (1985).

12. P. Pihet, S. Guldbakke, H.G. Menzel, H. Schuhmacher, Radiat. Prot. Dosim. 37, 1957 (1992).

13. M.J. Norgett, M.T. Robinson, I.T. Torrens, Nucl. Eng. Des. 33, 50 (1974).

14. 1992 Annual Books of ASTM Standards, Vol. 12.02, E521-89, E639-79 (1992).

15. J. Lindhard, V. Nielsen, M. Scharff, Mater. Fys. Medd. Dan. Vid. Selsk. 36 (1963).

16. D.G. Doran, N.J. Graves, HEDL-TME 7670, Hanford Engineering Development Laboratory (1976).

17. T. Fukahori et al., Reactor Dosimetry: Radiation Metrology and Assessment (ASTM STP 1398) (2001), p. 591.

18. N. Yamano et al., JAERI-1316, Japan Atomic Energy Institute (1989).

19. ICRP, 1990 publication 60 (Pergamon Press, Oxford, 1991).

20. T. Nakagawa et al., J. Nucl. Sci. Technol. 32, 1259 (1995).

21. T.W. Armstrong, K.C. Chandler, ORNL-4869, Oak Ridge National Laboratory (1973).

22. Y. Sakamoto et al., JAERI-1345, Japan Atomic Energy Institute (2003).

23. H. Schuhmacher, B.R.L. Siebert, Radiat. Prot. Dosim. 40, 85 (1992). 